\title{
Metodología de Diseño e Implementación de un Sistema para Generación de Campos Magnéticos Uniformes con Bobinas Helmholtz Cuadrada Tri-Axial
}

\author{
Andrés F. Restrepo ${ }^{(1)}$, Edinson Franco ${ }^{(1)}$ y Carlos R. Pinedo ${ }^{(2)}$ \\ Universidad del Valle, Escuela de Ingeniería Eléctrica y Electrónica, (1) Grupo de Investigación en Control \\ Industrial (GICl), (2) Grupo de Investigación en Percepción y Sistemas Inteligentes (PSI), Ciudad \\ Universitaria Meléndez, Calle 13 No. 100-00, Cali-Colombia \\ (e-mail: andres.restrepo@correounivalle.edu.co; edinson.franco@correounivalle.edu.co; \\ carlos.pinedo@correounivalle.edu.co)
}

Recibido Oct. 4, 2013; Aceptado Nov. 28, 2013; Versión final recibida Ene. 29, 2014

\section{Resumen}

Se presenta una metodología de diseño e implementación de un sistema para generación de campos magnéticos uniformes de baja magnitud y frecuencia, sobre un volumen específico utilizando una bobina Helmholtz cuadrada tri-axial. El procedimiento de diseño se basa en conceptos teóricos y simulaciones a partir de expresiones matemáticas simples que relacionan el área, con campo magnético uniforme, con la longitud de las bobinas. Además, se construye el modelo computacional y se simula usando software de elementos finitos. Se observa que los resultados teóricos son consistentes con los resultados del modelo de simulación y las pruebas mediante medición de campo magnético. Se concluye que la metodología de diseño permite establecer un procedimiento simplificado y repetible, para obtener campo magnético uniforme en un volumen de trabajo adecuado para realizar estudios in-vitro o in-vivo.

\section{A Design and Implementation Methodology of a System to Generate Uniform Magnetic Field Volume with Tri-axial Square Helmholtz Coils}

\begin{abstract}
The design and implementation methodology of a system to generate low-magnitude and low-frequency uniform magnetic fields on a specific volume using a tri-axial square Helmholtz coil is presented. The designing procedure is based on theoretical concepts and simulations from simple mathematical expressions that relate the area, with uniform magnetic field, with the length of the coils. Also, the computational model is constructed and simulated using finite element software. It is observed that theoretical results are consistent with simulation model results and the magnetic field measurement tests. It is concluded that the design methodology allows establishing a simplified and repeatable procedure to obtain a uniform magnetic field in the volume of interest suitable for in-vitro or in-vivo studies.
\end{abstract}




\section{INTRODUCCIÓN}

Existen numerosas aplicaciones de diferentes áreas de estudio donde se requiere la generación de campo magnético homogéneo y controlado, una de estas es la investigación en bioelectromagnetismo (De Seze et al., 1994). Muchos de los experimentos realizados para estudiar los efectos de la estimulación con campos magnéticos en sistemas biológicos requieren idealmente de dispositivos que puedan generar campos magnéticos homogéneos y que además garanticen una exposición controlada y repetible a las diferentes muestras involucradas en el experimento (Caputa y Stuchly, 1996). Una aplicación de campos magnéticos uniformes se realizó por Moncada (Moncada et al., 2011) para el crecimiento de osteoblastos. En el cual, por medio de un estudio clínico se obtuvieron menores tiempos de consolidación de fracturas en pacientes sometidos a estimulación magnética sobre la región afectada. Aunque estas diferencias en los tiempos de consolidación no fueron estadísticamente significantes, sirven como evidencia del efecto de los campos magnéticos en el crecimiento celular, tanto en el caso de proliferación como de inhibición de crecimiento. Este último comportamiento se evidencia en los estudios de Martino (Martino et al., 2010b), en el cual se someten células cancerígenas a bajos niveles de intensidad de campo magnético.

Para realizar un adecuado estudio de los efectos de la estimulación con campos magnéticos se debe determinar un volumen de trabajo donde se garantice una distribución de campo magnético uniforme; en este sentido, los arreglos de bobinas de núcleo de aire han sido tradicionalmente utilizados para generar una determinada distribución espacial de campo magnético, cuando estos son alimentados por una corriente eléctrica (Herceg et al., 2009). Por otro lado, los sistemas de control de campo magnético buscan compensar las componentes de campo magnético externo incluyendo el campo geomagnético (Martino et al., 2010b) que puedan interferir sobre la distribución de campo magnético en el volumen de trabajo deseado, esta compensación se puede realizar por medio de un sistema activo o pasivo (Choleris et al., 2002, Kellogg, 2003). La principal ventaja del sistema de compensación activa radica en que no solo se puede utilizar en aplicaciones de apantallamiento sino que también permite el ajuste de niveles de campo magnético controlado diferentes de cero dependiendo de la aplicación, esto es posible incorporando un sistema de medición y control al sistema de generación de campo magnético uniforme (Kellogg, 2003).

Teniendo en cuenta los requerimientos de las diferentes aplicaciones, la generación de campo magnético uniforme puede ser unidireccional — como en la mayoría de experimentos de crecimiento celular (Martino et al., 2010a) — o tridimensional — como por ejemplo en las pruebas de inmunidad magnética y generación de campo magnético rotatorio (Yongshun et al., 2012)_. Estos requerimientos junto a una adecuada metodología de diseño permiten definir tanto los parámetros físicos como eléctricos del sistema de irradiación. En este trabajo se presenta una metodología para el diseño y validación de un sistema de bobinas Helmholtz cuadradas triaxiales (HCT), para aplicaciones de estimulación con campos magnéticos de baja magnitud y frecuencia sobre sistemas biológicos in vivo e in vitro; se parte del diseño analítico de las bobinas a partir de expresiones matemáticas simples relacionando el área con campo magnético uniforme con la longitud de las bobinas, continuando con la validación mediante simulación del sistema usando software de cálculo con técnicas de elementos finitos y se finaliza presentando el diseño de los componentes y la validación experimental del campo magnético uniforme en el volúmen de interés.

El diseño fue dimensionado para realizar estudios que permitan la experimentación con placas de cultivo celular, pequeños animales o incluso pequeñas semillas. Considerando todos los parámetros de diseño se plantea un volumen esférico de radio $6 \mathrm{~cm}$ con campo magnético uniforme alrededor del centro de separación del sistema de bobinas HCT, el cual esta conformado por tres pares de bobinas Helmholtz cuadradas ortogonales entre sí con longitud media del lado $a_{1}=30 \mathrm{~cm}, a_{2}=29 \mathrm{~cm}$ y $a_{3}=28 \mathrm{~cm}$ respectivamente. Sin embargo, en los resultados experimentales se obtuvo un error de homogeneidad promedio menor a $0.45 \%$ para una distancia referida al centro de separación de $\pm 9 \mathrm{~cm}, \pm 8.7 \mathrm{~cm} \mathrm{y} \pm 7.8 \mathrm{~cm}$ sobre cada eje de simetría $(z, y, x)$.

\section{CAMPO MAGNÉTICO UNIFORME}

El estudio del comportamiento del campo magnético generado por las bobinas Helmholtz se puede realizar mediante la ley de Biot-Savart (Bell y Marino, 1989). La configuración de bobinas Helmholtz consiste en un par de bobinas circulares o cuadradas ubicadas de forma paralela una a cada lado del área experimental a lo largo de un eje común, separadas a una distancia $h$. Cada bobina está formada por $N$ vueltas de hilo conductor; para las bobinas cuadradas de longitud de lado $2^{\star}$ a como se ilustra en la Fig. 1, la relación entre el lado y la distancia de separación está dada por un factor de $0.5445\left(\mathrm{~h}=0.5445^{*} 2^{*} \mathrm{a}=1.089^{*} \mathrm{a}\right)$ (Tsz-Ka L., 2004). El par de bobinas se conecta en serie y por ellas circula una corriente eléctrica de intensidad $l$, generando un campo magnético que es prácticamente uniforme en el espacio comprendido entre ellas (Alamgir et al., 2005). 


\section{Bobinas Helmholtz cuadradas}

Para las bobinas Helmholtz cuadradas (Fig. 1), el campo magnético sobre el eje $z$ se calcula por medio de la ecuación (1).

$$
\vec{B}(z)=\frac{2 \mu_{0} N a^{2}}{\pi} *\left[\frac{1}{\left(a^{2}+\left(z+\frac{h}{2}\right)^{2}\right) *\left(2 a^{2}+\left(z+\frac{h}{2}\right)^{2}\right)^{1 / 2}}+\frac{1}{\left(a^{2}+\left(z-\frac{h}{2}\right)^{2}\right) *\left(2 a^{2}+\left(z-\frac{h}{2}\right)^{2}\right)^{1 / 2}}\right] \hat{z}
$$

Donde,

a = Longitud media del lado de la bobina

$\mathrm{h}=$ Distancia entre las bobinas.

z $\quad$ = Distancia entre el centro de separación y el punto evaluado.

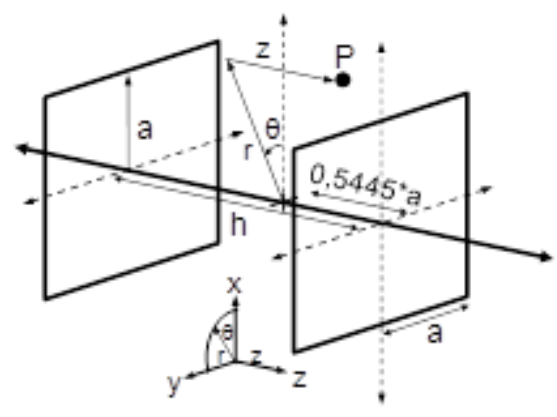

Fig. 1: Configuración de bobinas Helmholtz cuadradas

Para establecer el campo magnético en las bobinas, asumiendo que es constante y uniforme en el centro de ellas, la ecuación (1) se expresa en un segmento alrededor del centro $(z=0)$ mediante un desarrollo en series de Taylor, ecuación (2).

$\left.\mathrm{B}_{\mathrm{z}}(\mathrm{z})\right|_{|z| \leq a / 2} \approx \mathrm{B}_{\mathrm{z}}(\mathrm{z}=0)+\left.\frac{\partial \mathrm{B}_{\mathrm{z}}}{\partial \mathrm{z}}\right|_{\mathrm{z}=0} \mathrm{z}+\left.\frac{1}{2 !} * \frac{\partial^{2} \mathrm{~B}_{\mathrm{z}}}{\partial \mathrm{z}^{2}}\right|_{\mathrm{z}=0} \mathrm{z}^{2}+\left.\frac{1}{3 !} * \frac{\partial^{3} \mathrm{~B}_{\mathrm{z}}}{\partial \mathrm{z}^{3}}\right|_{\mathrm{z}=0} \mathrm{z}^{3}+\left.\frac{1}{4 !} * \frac{\partial^{4} \mathrm{~B}_{z}}{\partial \mathrm{z}^{4}}\right|_{\mathrm{z}=0} \mathrm{z}^{4}$

Realizando el desarrollo en series de Taylor de la ecuación (2) alrededor de $z=0$ se determina que por simetría $B_{z}(z)$ es una función par, lo que asegura que todas las derivadas impares de $B_{z}(z)$ son nulas. Resolviendo el término de la segunda derivada se obtiene la condición de uniformidad de las bobinas Helmholtz cuadradas (Tsz-Ka L., 2004) que relaciona la distancia de separación $h$ con la longitud media del lado de la bobina a $\left(\mathrm{h}=1.089^{\star} a\right)$, que al ser remplazada en (1) y evaluada en $z=0$, genera la expresión del campo magnético en el centro de separación de las bobinas cuadradas, ecuación (3) .

$$
\mathrm{B}_{\mathrm{z}}(0) \approx \frac{4 \mu_{0} \mathrm{NI}}{1.2965 \pi \sqrt{2.2965^{*} \mathrm{a}}}
$$

Con el propósito de encontrar la expresión equivalente en series de Taylor se calcula la cuarta derivada evaluada en $z=0$ (Restrepo-Alvarez et al., 2012). Finalmente, siempre y cuando se cumpla que $h=1.089^{*} a$, se obtiene la expresión del campo magnético en el intervalo $|z| \leq 0.5445^{\star} a, B_{z}(z)$, para la configuración de bobinas Helmholtz cuadradas (ecuación (4)).

$$
\left.\mathrm{B}_{\mathrm{z}}(\mathrm{z})\right|_{|z| \leq 0.5445 * \mathrm{a}} \approx \frac{4 \mu_{0} \mathrm{NI}}{1.2965 \pi \sqrt{2.2965} * \mathrm{a}} *\left[1-0.8068 *\left(\frac{\mathrm{z}}{\mathrm{a}}\right)^{4}\right]
$$

En términos generales, la distribución de campo magnético usando una configuración Helmholtz será homogénea solo para una determinada área ubicada en la mitad de la distancia de separación del par de bobinas (Torres O. et al., 2007).

\section{Especificación de homogeneidad de campo magnético}

La homogeneidad del campo magnético $(\mathrm{H})$ es una medida de la variabilidad del campo magnético dentro de 
una región definida del espacio (Wang et al., 2002), normalmente este valor se indica como una diferencia porcentual entre la magnitud de campo magnético en el punto de evaluación y la magnitud del campo magnético en el centro de separación de las bobinas, tal como se describe en la ecuación (5). El valor de homogeneidad de campo magnético depende de los requerimientos de la aplicación, los especificados con mayor frecuencia son los de $\pm 0.1 \%, \pm 0.5$ y $\pm 1 \%$, que son suficientes para la gran mayoría de casos (Marino, 2008). En la práctica se consideran volúmenes de forma cilíndrica, esférica y cúbica para especificar la homogeneidad del campo.

$$
H[\%]=\frac{B_{i}-B_{0}}{B_{0}} * 100
$$

Donde,

$\mathrm{B}_{0} \quad=$ Campo magnético en el centro de las bobinas.
$\mathrm{B}_{\mathrm{i}} \quad$ Campo magnético en otro punto dentro del volumen.

Remplazando la ecuación (1) en (5) se puede obtener la relación entre una longitud determinada con campo magnético uniforme (z) y la longitud media del lado de las bobinas (a). Sin embargo, la solución analítica completa resulta ser muy compleja, por lo que se plantea la solución de la ecuación evaluando diferentes valores de $z$, obteniendo una relación $z / a$ para determinados valores de homogeneidad. Finalmente, la expresión del campo magnético en el intervalo $|z| \leq 0.5445^{\star}$ a de la ecuación (4) permite obtener una relación aproximada de z/a como se muestra en la siguiente ecuación:

$$
|z| \leq\left(\frac{H}{80.68}\right)^{1 / 4} * a
$$

\section{BOBINAS HCT}

Este sistema es el encargado de la generación de campo magnético uniforme de baja magnitud y frecuencia el cual consta de un arreglo de tres pares de bobinas Helmholtz cuadradas, donde cada par se ubica en cada uno de los ejes coordenados ( $x, y, z)$ como se muestra en la Fig. 2. La geometría de bobinas cuadradas permite una mayor homogeneidad, mejor uso del volumen, facilidad de implementación y menor susceptibilidad a errores de construcción comparadas por ejemplo con las bobinas circulares (Restrepo-Alvarez et al., 2012).

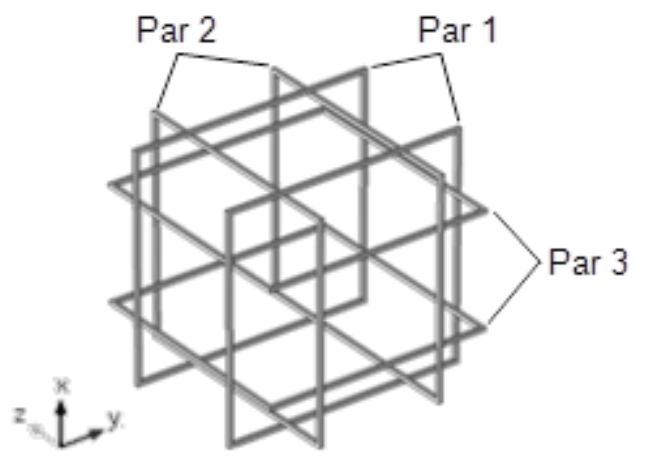

Fig. 2: Diagrama esquemático de las bobinas HCT

Las dimensiones de las bobinas $a_{i}$ y las distancias de separación $h_{i}$ dependen de las restricciones de la aplicación en cuanto al volumen de trabajo deseado con campo magnético uniforme. En este caso el diseño fue dimensionado para realizar estudios sobre sistemas biológicos in vivo e in vitro; es decir, que permita la experimentación con placas de cultivo celular, pequeños animales o incluso pequeñas semillas. La permeabilidad magnética de los materiales biológicos se considera similar a la permeabilidad magnética del vacío $\mu_{0}$ (Cabal et al., 2005). Sin embargo, debido a la concurrencia entre los campos magnéticos y eléctricos, es importante tener en cuenta las variables inducidas (campo eléctrico, densidad de corriente y calentamiento eléctrico) sobre el material biológico con el que se pretenda experimentar (Moncada et al., 2011).

Considerando los parámetros físicos de diseño se especifica un volumen esférico de radio $6 \mathrm{~cm}$ con campo magnético uniforme tomando como referencia la componente $B_{z}$ del par 1 de bobinas Helmholtz cuadradas. Según la relación de homogeneidad de ecuación (5), utilizando un par de bobinas cuadradas con longitud media del lado $a_{1}=30 \mathrm{~cm}$ se obtiene un máximo error de homogeneidad de aproximadamente $0.15 \%$ a lo largo del eje de simetría en el centro de separación. 


\section{Parámetros nominales}

Uno de los parámetros más importantes a considerar es la uniformidad del campo magnético generado por las bobinas Helmholtz debido a las limitaciones de frecuencia de operación, inductancia mutua y acople de impedancias; con el objetivo de especificar las limitaciones de operación se realiza la caracterización de las bobinas Helmholtz cuadradas utilizando estos parámetros de diseño (Cvetkovic y Cosic, 2007).

El cálculo de la resistencia $(R)$ de cada bobina está determinado por las ecuaciones (7) y (8), mientras que la inductancia se determina usando la ecuación (11), donde $\rho$ : resistividad del cobre $\left(\rho_{\text {cobre }}=1.7^{*} 10-8\left(\Omega^{*} \mathrm{~m}\right)\right.$ ), I : longitud del alambre, $s$ : sección transversal del alambre, $\mathrm{d}$ : diámetro del alambre, $\mathrm{N}$ : número de vueltas, $D$ : longitud del lado de bobina $\left(D=2^{*} a(m)\right)$ y $\alpha$ : factor de inductancia mutua $\left(0.494^{*} 10^{6}\right)$.

$$
\begin{aligned}
& R=\frac{\rho l}{s}=\frac{4 \rho l}{\pi d^{2}} \\
& I=\frac{R \pi d^{2}}{4 \rho}=4 N D
\end{aligned}
$$

Una de las limitaciones básicas en cuanto a la frecuencia de operación es el tiempo de subida del campo magnético en la bobina ( $\tau$ ). Este tiempo se calcula usando la ecuación (9) y corresponde al valor entre el $10 \%$ y $90 \%$ de la máxima intensidad de campo magnético.

$$
\tau(10 \%-90 \%)=2.2 * \frac{L}{R}
$$

La inductancia serie de las bobinas se puede calcular mediante la ecuación (10) (Javor y Anderson, 1998). La inductancia mutua $(M)$ se define entre cada par de bobinas y se determina por medio de $M=\alpha^{*} N^{2 * a}$ (Bronaugh, 1995). Es decir, que no se tiene en cuenta el acople de los otros dos pares de bobinas.

$$
\mathrm{L}=\frac{2 \mu_{0} \mathrm{DN}}{\pi} *\left[\ln \left(\frac{2 \mathrm{D}}{\mathrm{d}}\right)-0.774\right]
$$

De modo que, la inductancia resultante $\left(\mathrm{L}_{\mathrm{r}}\right)$ para un par de bobinas está determinada por la ecuación (11).

$$
L_{r}=L+M=\frac{4 \mu_{0} D N^{2}}{2 \pi} *\left[\ln \left(\frac{2 D}{d}\right)-0.465\right]
$$

En condiciones ideales de operación, la frecuencia máxima no debe ser mayor a $0.5^{\star} \tau_{\mathrm{r}}$ para garantizar que no se presenten pérdidas en la intensidad del campo magnético. Teniendo en cuenta los parámetros eléctricos de diseño y considerando una frecuencia máxima de operación hasta $100 \mathrm{~Hz}$, se define el diámetro del conductor de cobre y el número de vueltas para el juego de bobinas con el objetivo de calcular los parámetros de resistencia e inductancia. Por lo tanto, para cada par de bobinas cuadradas con un número de vueltas $\mathrm{N}=50$ y un conductor de cobre calibre 20 AWG clase térmica $200{ }^{\circ} \mathrm{C}\left(I_{\text {máx }}=5.1 \mathrm{~A}\right)$ con diámetro

\begin{tabular}{|c|c|c|c|c|}
\hline \multicolumn{2}{|c|}{ Parámetro } & Par 1 & Par 2 & Par 3 \\
\hline \multicolumn{2}{|c|}{ Longitud media del lado de la bobina (a) } & $0.30 \mathrm{~m}$ & $0.29 \mathrm{~m}$ & $0.28 \mathrm{~m}$ \\
\hline \multicolumn{2}{|c|}{ Distancia de separación $(\mathrm{h})$} & $0.3267 \mathrm{~m}$ & $0.3158 \mathrm{~m}$ & $0.3049 \mathrm{~m}$ \\
\hline \multicolumn{2}{|c|}{ Corriente máxima a aplicar (Imáx) } & $5.1 \mathrm{~A}$ & $5.1 \mathrm{~A}$ & $5.1 \mathrm{~A}$ \\
\hline \multicolumn{2}{|c|}{ Campo magnético según $I_{\text {máx }}(B)$} & $692.2 \mu \mathrm{T}$ & $716.1 \mu \mathrm{T}$ & $741.7 \mu \mathrm{T}$ \\
\hline \multirow{2}{*}{$\begin{array}{c}\text { Resistencia de la } \\
\text { bobina (R) }\end{array}$} & Calculado & $7.91 \Omega$ & $7.64 \Omega$ & $7.38 \Omega$ \\
\hline & Medido & $7.90 \Omega$ & $7.65 \Omega$ & $7.75 \Omega$ \\
\hline \multirow{2}{*}{$\begin{array}{l}\text { Inductancia de la } \\
\text { bobina }\left(L_{r}\right)\end{array}$} & Calculado & $8.20 \mathrm{mH}$ & $7.89 \mathrm{mH}$ & $7.57 \mathrm{mH}$ \\
\hline & Medido & $10.64 \mathrm{mH}$ & $10.31 \mathrm{mH}$ & $10.81 \mathrm{mH}$ \\
\hline
\end{tabular}
nominal $\mathrm{d}=0.813 \mathrm{~mm}$ y resistividad $\rho=1.7^{*} 10^{-8} \Omega^{*} \mathrm{~m}$, se obtienen los parámetros nominales calculados tanto físicos como eléctricos que se presentan en la Tabla 1. Además, se muestran los valores medidos de resistencia e inductancia para cada par de bobinas Helmholtz.

Tabla 1: Parámetros nominales de las bobinas HCT 
Considerando aplicaciones de generación de campo magnético de baja magnitud y frecuencia (Novikov et al., 2009) en el orden del campo geomagnético cuya magnitud dependiendo de la ubicación geográfica oscila entre $25 \mu \mathrm{T}$ y $65 \mu \mathrm{T}$ (Martino et al., 2010b), se define un rango de generación de $\pm 200 \mu T$ para cada componente de campo magnético $B_{z}, B_{y}, y B_{x}$; es decir, que la corriente máxima de operación para cada par de bobinas será $1.5 A, 1.45 A$ y $1.4 A$ respectivamente. Este sistema permite la generación de campos magnéticos estáticos y variantes en el tiempo (formas de onda senoidal, cuadrada y triangular), teniendo en cuenta que la frecuencia máxima de operación es de $100 \mathrm{~Hz}$.

Para obtener los valores medidos que se registran en la Tabla 1, se utilizó un multímetro digital MINIPA modelo ET-2110. En este proceso se realizaron, para cada par de bobinas, cuatro mediciones instantáneas y el promedio entre ellas fue el que finalmente se tomó como el valor medido. Es importante aclarar que el valor de corriente máxima a aplicar $I_{\text {máx }}=5.1 \mathrm{~A}$, se refiere a la máxima corriente que soporta el conductor de cobre calibre 20 AWG clase térmica $200^{\circ} \mathrm{C}$ y no a la corriente máxima de operación. Estos valores de corriente de operación son relativamente bajos con respecto a $I_{\text {máx }}$ lo que minimiza la ocurrencia de un calentamiento significativo en las bobinas y por ende en la región de trabajo. Los valores medidos de resistencia presentan una buena correlación con los datos calculados, el error que se presenta en el par 3 puede ser debido a empalmes en el conductor realizados en el proceso de construcción de la bobina. Por otro lado, las mediciones de inductancia presentan un error significativo con los valores calculados debido a que el modelo utilizado considera solo la inductancia mutua entre los arrollamientos de cada par de bobinas y no tiene en cuenta el acople con los otros dos pares de bobinas.

\section{Modelo computacional}

Con el objetivo de verificar la distribución y uniformidad de cada componente de campo magnético $B_{z}$, $B_{y}$, y $B_{x}$ sobre el volumen de trabajo, se diseñó en COMSOL ${ }^{\circledR}$ el modelo computacional 3D que se muestra en la Fig. 3. El modelo de las bobinas HCT está conformado por tres pares de bobinas Helmholtz cuadradas y un cubo suficientemente grande al cual se asigna el potencial cero para hacer el análisis electromagnético (Olivares-Galvan et al., 2010).

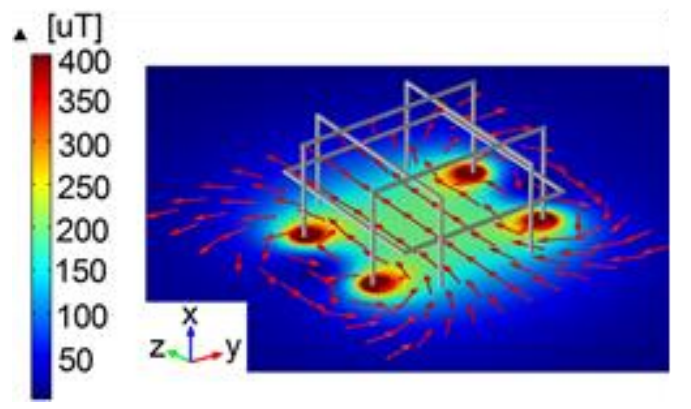

a) Par $1-B_{z}$

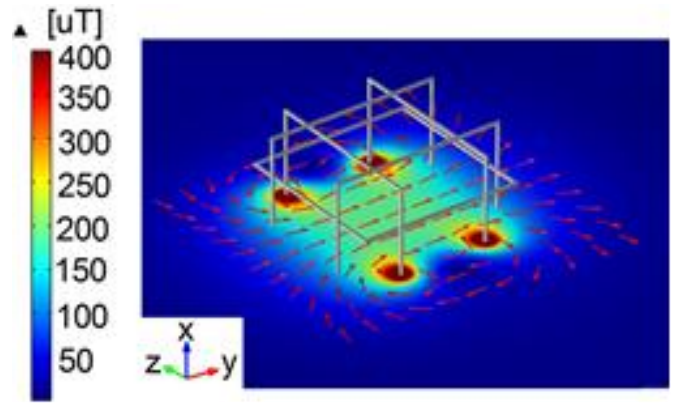

b) Par 2 - $B_{y}$

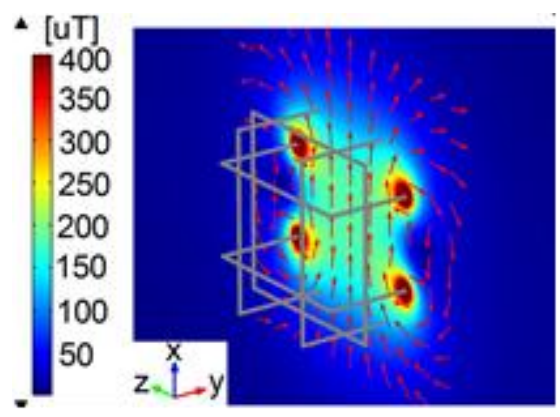

c) Par 3 - $B_{x}$

Fig. 3: Simulación de líneas de campo magnético y área uniforme de las bobinas HCT

Teniendo en cuenta los parámetros de diseño (físicos y eléctricos) y las condiciones de operación del sistema de bobinas HCT, se construyó el modelo y se realizó la simulación en el punto de operación de $200 \mu \mathrm{T}$ energizando cada par de bobinas independientemente con una corriente constante de $1.5 \mathrm{~A}$, $1.45 \mathrm{~A}$ y $1.4 \mathrm{~A}$ respectivamente. Para el análisis electromagnético se utilizaron básicamente las propiedades físicas y eléctricas del conductor (área transversal, calibre, conductividad, resistividad, corriente) y los diferentes parámetros geométricos de las bobinas (longitud, número de vueltas). En este caso de estudio no fueron considerados valores de permitividad (por trabajar en condiciones cuasi-estáticas) y el valor de permeabilidad relativa usada fue de 1 (permeabilidad del vacío) para 
condiciones de simulación sin material biológico. En esta etapa de la simulación se deben tener en cuenta una vez definido el tipo de sistema biológico a tratar, las variables eléctricas tanto del material biológico como de los elementos sobre cual se va a ubicar en el volumen de interés (placas de cultivo celular, bases de acrílico, etc.) (Moncada et al., 2010).

Una vez construida la geometría y asignadas las propiedades eléctricas, se realizó la caracterización de las condiciones de carga y mallado para el análisis electromagnético (el programa COMSOL utiliza el método de los elementos finitos para la solución de las ecuaciones de Maxwell). Cada par de bobinas Helmholtz (consideradas los elementos fuente) se caracterizó como un material conductor sólido alimentado por voltaje sin considerar efectos de corrientes Eddy. El modelo es implementado usando el módulo AC/DC de COMSOL para hacer el análisis de campos magnéticos estáticos y de baja frecuencia. El modelo computacional permite evaluar diferentes condiciones de carga.

En la Fig. 3 se muestran los mapas de contorno de densidad de flujo magnético para cada par de bobinas. Además, se puede observar la dirección de las líneas de campo magnético y el área de uniformidad sobre el centro de separación de las bobinas. Las magnitudes de los niveles de campo magnético se expresan en unidades de microtesla $(\mu \mathrm{T})$. Los resultados de simulación permiten verificar la uniformidad del campo magnético estático de aproximadamente $190 \mu \mathrm{T}$ en el centro de separación de cada par de bobinas, energizando cada par independientemente con una corriente constante de 1.5 A, 1.45 A y 1.4 A respectivamente. El error de homogeneidad obtenido en simulación es menor que $0.15 \%$ para una distancia de aproximadamente $\pm 6.2 \mathrm{~cm}$ a lo largo del eje de simetría con respecto al centro de separación. Esto para cada uno de los ejes coordenados (x, y, z).

Por otra parte, en los resultados experimentales se obtuvo una magnitud de campo magnético estático promedio de $204 \mu \mathrm{T}$ medido en el centro de separación de cada par de bobina. El error máximo encontrado entre los valores medidos y simulados fue de $7.4 \%$, este error está asociado en parte a la incertidumbre del proceso de medición (condiciones ambientales, resolución de equipos de medición, etc.) y a los métodos numéricos (aproximaciones matemáticas) que utiliza el programa de simulación para encontrar la solución al problema.

\section{Implementación del sistema.}

Una vez verificada la distribución y uniformidad del campo magnético en el sistema de bobinas propuesto, se inicia la etapa de construcción del mismo. La estructura para el arrollamiento del conductor debe ser construido de un material rígido con permeabilidad magnética similar a la del vacío, es decir que no presente alteraciones ante campos magnéticos externos. Además, se debe tener en cuenta el diámetro del conductor y el número de vueltas de la bobina para definir un volumen adecuado para el arrollamiento del conductor.

Finalmente, la estructura se construyó usando un material denominado poliestireno de alta densidad que cumple con las propiedades de rigidez y permeabilidad magnética suficientes para la implementación del sistema. Considerando un conductor 20 AWG con $\mathrm{d}=0.813 \mathrm{~mm}$ y $\mathrm{N}=50$, se plantea un arrollamiento de siete capas donde la primer capa es de ocho vueltas y las restantes de siete, definiendo entonces un espacio nominal de $1 \mathrm{~cm} \times 1 \mathrm{~cm}$ como se muestra en la Fig. 4a. Este arreglo permite obtener una sección transversal cuadrada en el arrollamiento completo de cada bobina.

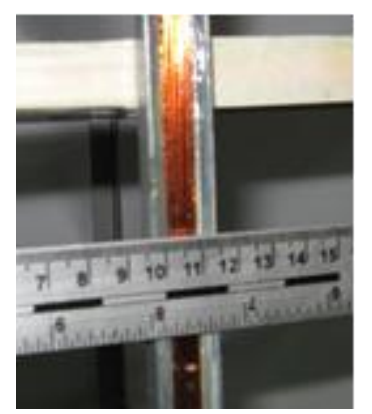

a) Estructura para el arrollamiento del conductor

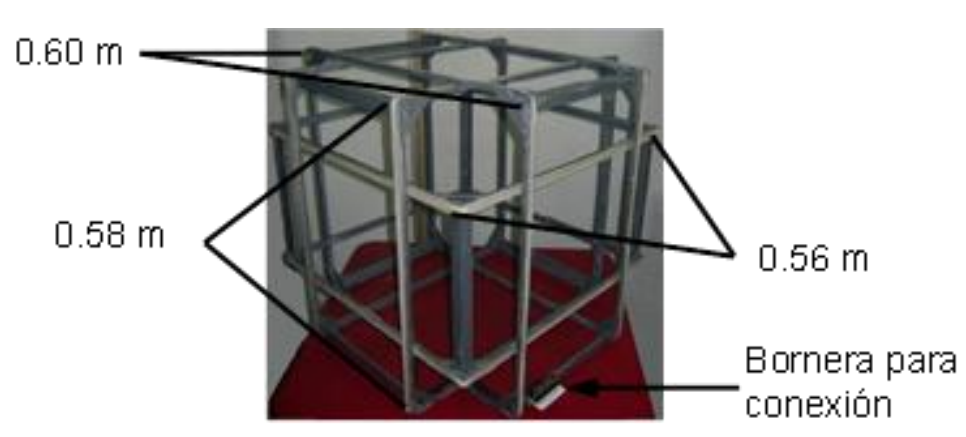

b) Sistema completo

Fig. 4: Implementación de las bobinas HCT

Según los parámetros de longitud de la Tabla 1 se construye cada par de bobinas cuadradas independientemente y posteriormente se realiza el proceso de ensamble, dando como resultado la estructura del sistema completo que se muestra en la Fig. $4 b$. 


\section{MEDICIÓN DE LA DISTRIBUCIÓN DE CAMPO MAGNÉTICO EN LAS BOBINAS HCT}

La intensidad de campo magnético se mide usando el sensor de efecto Hall lineal AD22151, cuya salida es un voltaje proporcional al campo magnético aplicado perpendicularmente a la superficie del empaquetado. Dentro de las principales características este sensor incluye la cancelación de compensación dinámica de deriva y un sensor de temperatura incorporado. Una vez especificados los parámetros de operación de los sensores, estos se ubican ortogonalmente entre sí con el propósito de obtener la magnitud del campo magnético en cada uno de los ejes coordenados $\left(B_{x}, B_{y}, B_{z}\right)$ (Hanna et al., 2009).

Para la validación del sensor se utilizó el equipo de medición de campo magnético FW Bell modelo 5170 propiedad del laboratorio de Alta Tensión de la Universidad del Valle, el cual cuenta con las calibraciones requeridas según las normas correspondientes. Para las pruebas experimentales del sistema se utilizó una fuente de voltaje GWINSTEK modelo GPC-3030D, un multímetro digital MINIPA modelo ET-2110 y un osciloscopio RIGOL modelo DS1202CA. El sistema de potencia encargado de energizar las bobinas HCT tiene la capacidad de suministrar $\pm 25 \mathrm{~V} @ 3.5 \mathrm{~A}$. Los resultados experimentales se obtuvieron para condiciones de operación sin material biológico.

La medición de la distribución de campo magnético se realiza sobre el eje de simetría de cada par de bobinas y en el volumen alrededor del centro de separación de las mismas (Azpúrua, 2012); para llevar a cabo estas mediciones es necesario determinar la ubicación espacial exacta de los sensores en cada uno de los ejes coordenados $(x, y, z)$ con el objetivo de realizar un mapeo del campo magnético en diferentes puntos alrededor del volumen de trabajo.

Por lo tanto, se plantea la construcción y uso de una estructura base donde se ubica el sistema de medición y que permite el desplazamiento en todas las direcciones $(x, y, z)$ alrededor del volumen ubicado en el centro de separación de las bobinas HCT. La estructura base se construye de un material rígido cuya permeabilidad magnética no presenta alteraciones ante campos magnéticos externos, en este caso se utiliza PVC para la implementación. El arreglo experimental se muestra en la Fig. 5, donde se puede observar la estructura base Fig. 5a y los sensores en configuración tri-axial Fig. $5 \mathrm{~b}$.

Inicialmente, para caracterizar cada par de bobinas se realiza la medición de campo magnético estático ubicando el sensor exactamente en el centro de separación las bobinas HCT y energizando cada par de bobinas independientemente con diferentes valores de corriente. Posteriormente, fijando un punto de operación con un valor constante de corriente en cada par de bobinas, se realiza la medición de campo magnético sobre cada eje de simetría con el propósito de determinar la distribución de campo magnético. Finalmente, estos valores medidos se comparan con los datos calculados para cada par de bobinas HCT. Todas las pruebas experimentales se realizan considerando la posición del sistema de bobinas HCT en el sistema de coordenadas ( $x, y, z)$ como se muestra en la Fig. 2.

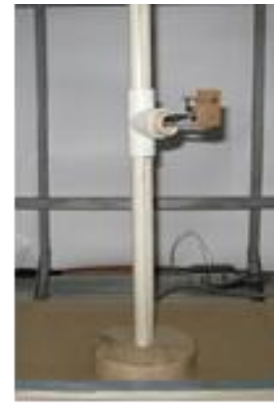

a) Estructura base

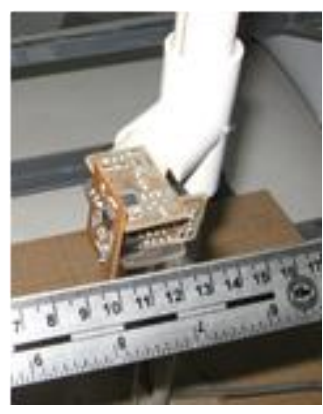

b) Sensores

Fig. 5: Arreglo experimental para determinar la distribución de campo magnético

\section{Campo magnético en el centro de separación.}

El comportamiento estático de las bobinas HCT se analiza a partir de la curva característica que representa el campo magnético medido con el sensor como una función de la corriente que circula por el par de bobinas. En la Fig. 6 se presentan los resultados del campo magnético medido en el centro de separación de cada par de bobinas ante variaciones de la corriente aplicada en el rango de -1.6 A y $1.6 \mathrm{~A}$. Los resultados muestran una alta linealidad en cada par de bobinas y una buena correlación entre los datos calculados y medidos. Además, se puede observar que en el par 3 de bobinas se obtiene una mayor magnitud de campo magnético comparada con los otros dos pares. Esto se debe a que el par 3 de bobinas es la de menor longitud de lado y por consiguiente al obtener el campo magnético evaluando el mismo valor de corriente aplicada, se obtiene por 
medio de la ecuación (1), que el campo magnético generado es inversamente proporcional a la longitud del lado de la bobina. Finalmente, es importante aclarar que los valores de campo magnético negativos presentados en las curvas corresponden al cambio en el sentido de la corriente eléctrica cuando esta se hace menor a cero.

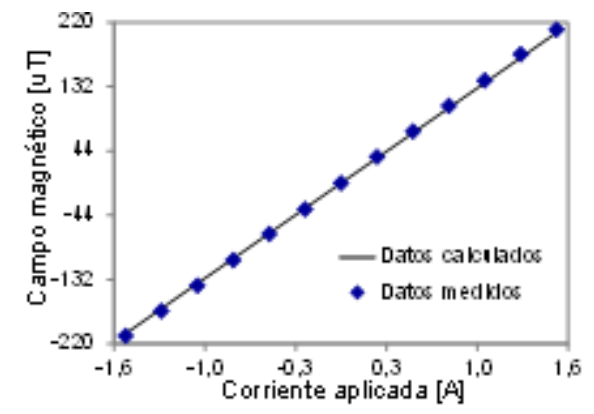

a) Par 1 - $B_{z}$

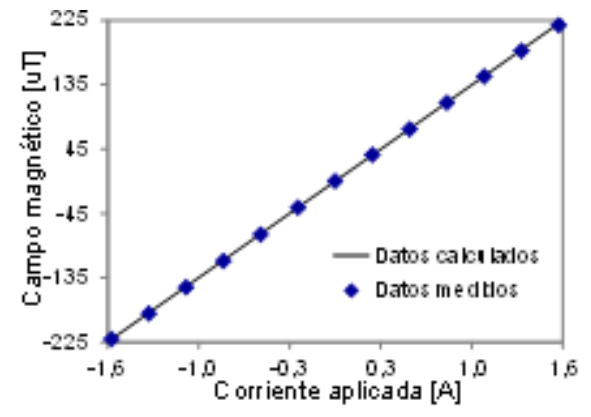

b) Par 2 - $\mathrm{B}_{\mathrm{y}}$

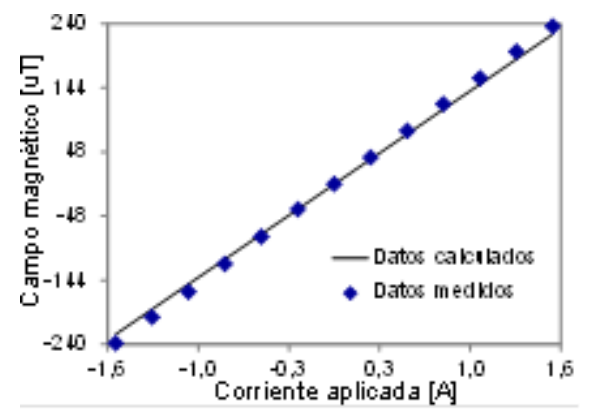

c) Par 3 - $B_{x}$

Fig. 6: Curvas características del comportamiento estático de las bobinas HCT

En el campo magnético medido con los sensores se encontró un error máximo de $6.48 \%$ con respecto al valor calculado para el par 3 de la componente $B_{x}$. Además, se obtuvo un error máximo de $1.5 \%$ con respecto al campo magnético medido usando el equipo FW Bell 5170 que se mencionó anteriormente.

Campo magnético sobre el eje de simetría.

El procedimiento experimental que permite determinar la distribución de campo magnético estático sobre cada eje de simetría del sistema de bobinas HCT involucra el uso de la estructura base para realizar el desplazamiento de los sensores sobre cada eje de simetría cuando se energice el par de bobinas correspondiente.

Considerando que la distribución de campo magnético usando una configuración Helmholtz es homogénea solo para una determinada área ubicada alrededor del centro de separación del par de bobinas, se ubica el sensor exactamente en el centro del volumen de interés y define un desplazamiento máximo del sensor de $\pm 15 \mathrm{~cm}$ respecto al centro de separación sobre el eje de simetría correspondiente. El desplazamiento del sensor se hace cada $1 \mathrm{~cm}$ donde se realiza la respectiva medición de campo magnético. Durante las pruebas experimentales cada par de bobinas se energizó de manera independiente con I = $1.5 \mathrm{~A}$.

En la Fig. 7 se observa la distribución de campo magnético normalizado $\left(B_{(i)} / B_{\text {máx }}\right)$ en función de la distancia de separación $\left(\mathrm{z}_{(\mathrm{i})}\right)$, según los datos calculados usando la ecuación (1) y los datos medidos experimentalmente. De los resultados se obtiene un error de homogeneidad promedio menor a $0.45 \%$ para una distancia referida al centro de separación de $\pm 9 \mathrm{~cm}, \pm 8.7 \mathrm{~cm}$ y $\pm 7.8 \mathrm{~cm}$ sobre cada eje de simetría $(z, y, x)$. En los resultados se puede observar que el mayor valor de $B_{\text {máx }}$ corresponde al medido en el par 3 de bobinas, esto tiene que ver como se mencionó anteriormente con la diferencia de longitud de lado de los pares de bobinas, las cuales se energizan al mismo valor de corriente eléctrica $(1.5 \mathrm{~A})$.

La resolución del campo magnético medido está limitada principalmente por la resolución de los equipos utilizados en la medición de las variables eléctricas. Por ejemplo, para medir el voltaje de salida del sensor utilizando un multímetro con resolución de $0.001 \mathrm{~V}$, se obtiene que el cambio mínimo de campo magnético que se puede medir es de aproximadamente $996 \mathrm{nT}$. Por lo tanto, para verificar el error de homogeneidad especificado en el diseño de $0.15 \%$ con respecto al valor de campo magnético de $218 \mu \mathrm{T}$, se debe contar con un sistema de medición cuya resolución máxima sea de aproximadamente 327 nT. Sin embargo, 
teniendo en cuenta esta limitación, los resultados experimentales muestran que el campo magnético sobre cada uno de los ejes de simetría se mantiene constante para una distancia referida al centro de separación de $\pm 6 \mathrm{~cm}$, validando el comportamiento esperado del sistema de bobinas HCT.

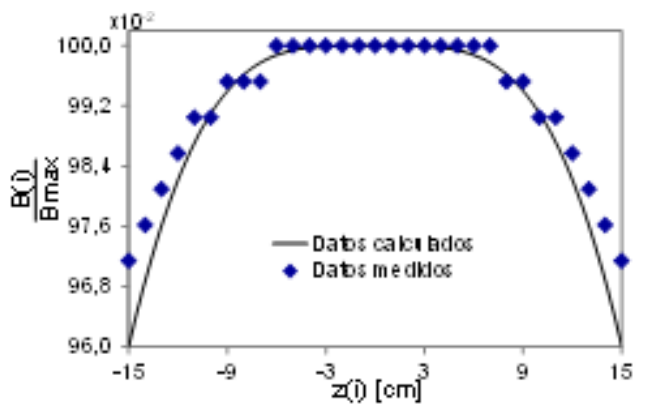

a) Par 1, $B_{\text {máx }}=206 \mu T$

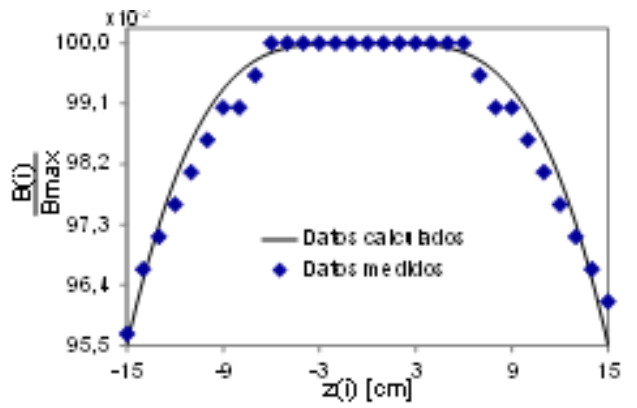

b) Par 2, $B_{\text {máx }}=212 \mu \mathrm{T}$

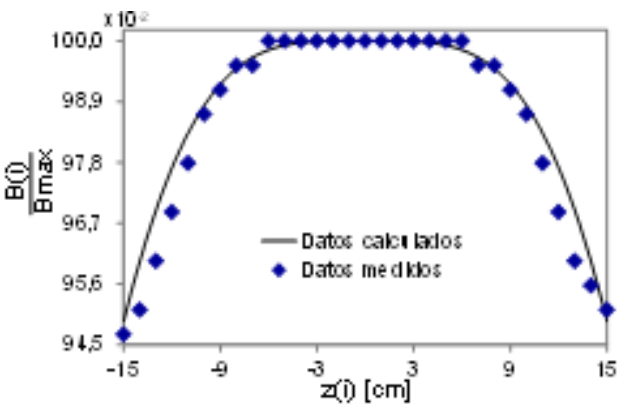

c) Par 3, $B_{\text {máx }}=219 \mu T$

Fig. 7: Distribución de campo magnético medido sobre los ejes de simetría de las bobinas HCT

Campo magnético sobre el volumen de trabajo.

Utilizando la estructura base para el desplazamiento de los sensores, se realiza la medición de campo magnético en diferentes puntos alrededor del centro de separación de las bobinas HCT con el objetivo de determinar la distribución del campo magnético sobre el volumen de trabajo. Inicialmente se plantea la construcción de mapas de contorno 2D por medio de la medición de campo magnético en diferentes puntos sobre un área de $13 \mathrm{~cm} \times 13 \mathrm{~cm}$ alrededor de centro de separación, formando un plano sobre el eje simetría de cada par de bobinas. La distancia entre los puntos de medición se definió de $1 \mathrm{~cm}$.
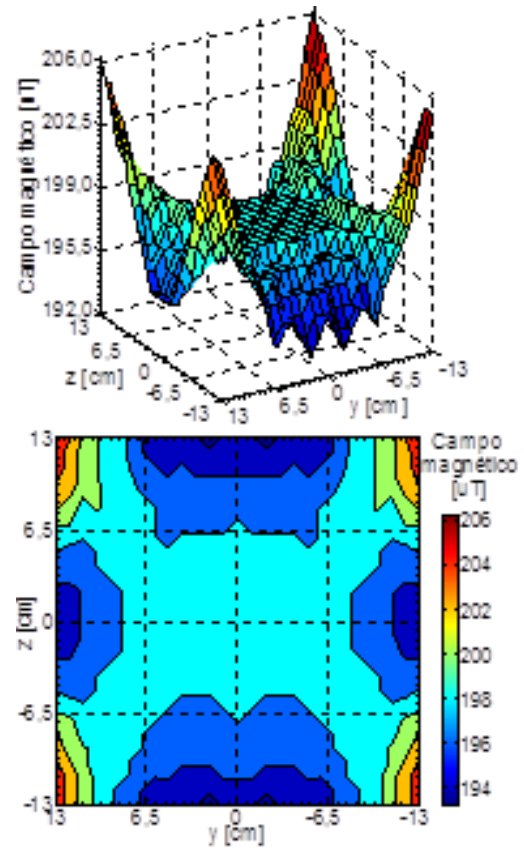

a) Par 1, plano yz $(x=0)$
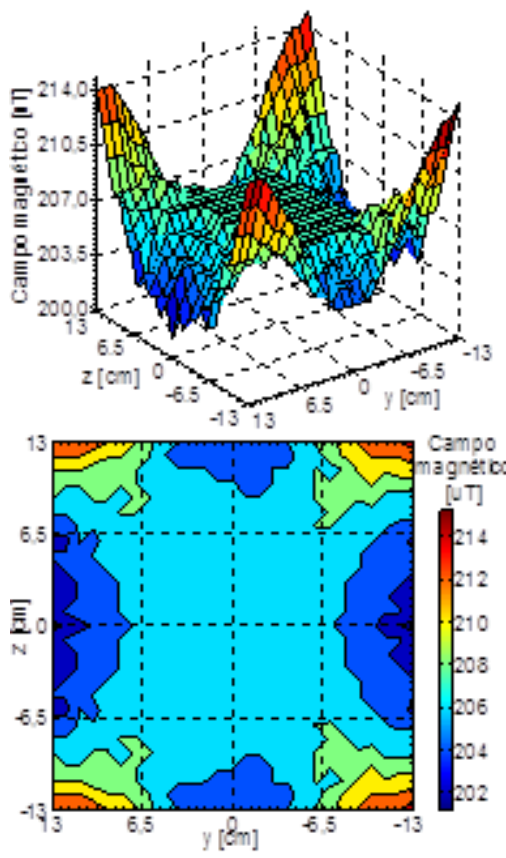

c) Par 2, plano yz $(x=0)$
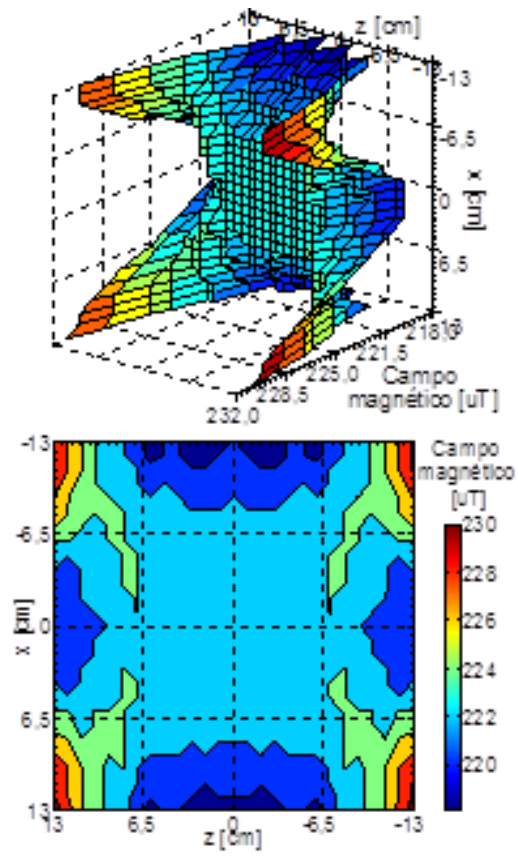

e) Par 3, plano zx $(y=0)$

Fig. 8: Distribución de campo magnético estático medido en las bobinas HCT 
Durante las pruebas experimentales cada par de bobinas se energizó independientemente con una corriente constante de 1.5 A. En la Fig. 8 se presentan los diagramas tridimensionales de la magnitud del campo magnético y los mapas de contorno 2D de la distribución de campo magnético estático para el punto de operación evaluado (1.5 A) en cada par de bobinas del sistema HCT. En los diagramas se observan picos de campo magnético en las esquinas, esto se debe a que la intensidad de campo magnético es mayor en la región más cercana al conductor. Estos resultados permiten verificar que alrededor del centro de separación se obtiene una buena uniformidad como lo muestran los mapas de contorno 2D.

Los resultados muestran una distribución homogénea del campo magnético en una área aproximada de 12 $\mathrm{cm} \times 12 \mathrm{~cm}$ sobre cada uno de los planos de simetría del sistema de bobinas HCT, obteniendo un volumen con campo magnético uniforme alrededor del centro de separación. Estos resultados experimentales fueron verificados con los resultados de simulación obtenidos por medio de la herramienta computacional COMSOL ${ }^{\circledR}$. El proceso de simulación permite obtener una solución más amplia del comportamiento del campo magnético en el sistema de bobinas HCT y considerar diferentes condiciones de operación con el objetivo de prever el comportamiento real del sistema.

\section{CONCLUSIONES}

De los resultados mostrados se concluye que para obtener un campo magnético uniforme de baja magnitud y frecuencia sobre cada uno de los ejes coordenados alrededor del centro de separación, se pueden emplear bobinas Helmholtz cuadradas tri-axiales ubicando cada par en cada uno de los ejes coordenados (x, y, z); la característica principal de esta metodología de diseño es la utilización de la relación obtenida por series de Taylor entre homogeneidad y longitud del lado de cada par de bobinas, lo que permite definir el volumen efectivo con campo magnético uniforme y simplificar los cálculos, permitiendo por un lado hacer más sencillo el diseño y por otro facilitar el proceso de diseño reproducible para diferentes aplicaciones; con esta metodología se concluye que el diseño puede ser validado por simulación empleando un modelo de computación 3D por medio del método de elementos finitos FEM — para verificar la distribución de campo magnético alrededor del centro de separación ante diferentes condiciones de operación del sistema de bobinas HCT-y/o usando un método de medición de la distribución de campo magnético alrededor del centro de separación de las bobinas HCT basado en la medición de diferentes puntos sobre el volumen de trabajo por medio de una estructura base para determinar la ubicación exacta de los sensores. Finalmente se concluye que los resultados del diseño son consistentes con los teóricos.

\section{AGRADECIMIENTOS}

Los autores agradecen al Programa Jóvenes Investigadores e Innovadores "Virginia Gutiérrez de Pineda" de COLCIENCIAS. Además, a la Vicerrectoría de Investigaciones por la financiación de este trabajo a través del proyecto Cl 2657, al Programa de Posgrados en Ingeniería Eléctrica y Electrónica (PPIEE) y a los Grupos de Investigación "Farmacología Univalle", "GICI", "PSI" y "GRALTA" de la Universidad del Valle.

\section{REFERENCIAS}

Alamgir, A. K. M., Fang, J., Gu, C. y Han, Z., Square Helmholtz coil with homogeneous field for magnetic measurement of longer HTS tapes. Physica C: Superconductivity, 424(1-2), 17-24 (2005).

Azpúrua, M. A., Estimación de la Incertidumbre en la Medición de la Homogeneidad Espacial del Campo Magnético en Sistemas de Generación de Campo Magnetostático utilizando el Método de Monte Carlo, 5to Congreso Iberoamericano de Estudiantes de Ingeniería Eléctrica (V CIBELEC 2012), 1-8, Mérida, Venezuela, 7-11 Mayo (2012).

Bell, G. B. y Marino, A. A., Exposure System for Production of Uniform Magnetic Fields. Electromagnetic Biology and Medicine, 8(2), 147-158 (1989).

Bronaugh, E. L., Helmholtz coils for calibration of probes and sensors: limits of magnetic field accuracy and uniformity, Electromagnetic Compatibility, 1995. IEEE International Symposium on, 72-76, Atlanta, United States, 14-18 Aug. (1995).

Cabal, C., Otero, G. y Acuña, J., Informe sobre Campos electromagnéticos y la salud humana, Universidad de la República, Montevideo, Uruguay, 29 p. (2005).

Caputa, K. y Stuchly, M. A., Computer controlled system for producing uniform magnetic fields and its application in biomedical research. Instrumentation and Measurement, IEEE Transactions on, 45(3), 701-709 (1996). 
Cvetkovic, D. y Cosic, I., Modelling and Design of Extremely Low Frequency Uniform Magnetic Field Exposure Apparatus for In Vivo Bioelectromagnetic Studies, Engineering in Medicine and Biology Society, 29th Annual International Conference of the IEEE, 1675-1678, Lyon, France, 22-26 Aug. (2007).

Choleris, E., Del Seppia, C., Thomas, A. W., Luschi, P., Ghione, S., Moran, G. R. y Prato, F. S., Shielding, but not zeroing of the ambient magnetic field reduces stress-induced analgesia in mice. Proceedings of the Royal Society of London. Series B: Biological Sciences, 269(1487), 193-201 (2002).

De Seze, R., Lahitte, A., Moreau, J. M. y Veyret, B., Generation of extremely-low frequency magnetic fields with standard available commercial equipment: Implications for experimental bioelectromagnetics work. Bioelectrochemistry and Bioenergetics, 35(1-2), 127-131 (1994).

Hanna, S. A., Motai, Y., Varhue, W. J. y Titcomb, S., Very-Low-Frequency Electromagnetic Field Detector With Data Acquisition. Instrumentation and Measurement, IEEE Transactions on, 58(1), 129-140 (2009).

Herceg, D., Juhas, A. y Milutinov, M., A Design of a Four Square Coil System for a Biomagnetic Experiment. Facta Universitatis Series : Electronics and Energetics, 22(3), 285-292 (2009).

Javor, E. R. y Anderson, T., Design of a Helmholtz coil for low frequency magnetic field susceptibility testing, Electromagnetic Compatibility 1998, IEEE International Symposium on, 912-917, Denver, United States, 2428 Aug. (1998).

Kellogg, J., Magnetic active compensation system (MACS) for electron microscopy, ETS-Lindgren, Interference Technology - Annual Guide, United States, 4 p., December (2003).

Marino, A. R., Homogeneidad de campo en bobinas de Helmholtz (NASO2-e-2), Serviciencia, S. L., Toledo, España, 4 p., Octubre (2008).

Martino, C. F., Perea, H., Hopfner, U., Ferguson, V. L. y Wintermantel, E., Effects of Weak Static Magnetic Fields on Endothelial Cells. Bioelectromagnetics, 31(4), 296-301 (2010a).

Martino, C. F., Portelli, L., McCabe, K., Hernandez, M. y Barnes, F., Reduction of the Earth's Magnetic Field Inhibits Growth Rates of Model Cancer Cell Lines. Bioelectromagnetics, 31(8), 649-655 (2010b).

Moncada, M. E., Pinedo, C. R., Criollo, W. D., Gutiérrez, J. O. y Cadavid, H., Modelo computacional 3D para estimar variables eléctricas inducidas en un cultivo in vitro estimulado por campos magnéticos de baja frecuencia. Ingeniería y Competitividad, 12(2), 69-79 (2010).

Moncada, M. E., Sarmiento, C., Martinez, C. y Martinez, A., Magnetic stimulation for fracture consolidation Clinical study. Engineering in Medicine and Biology Society EMBC 2011 Annual International Conference of the IEEE, 1141-1144, Boston, USA. (2011).

Novikov, V. V., Novikov, G. V. y Fesenko, E. E., Effect of weak combined static and extremely low-frequency alternating magnetic fields on tumor growth in mice inoculated with the Ehrlich ascites carcinoma. Bioelectromagnetics, 30(5), 343-351 (2009).

Olivares-Galvan, J. C., Campero-Littlewood, E., Escarela-Perez, R., Magdaleno-Adame, S. y Blanco-Brisset, E., Coil Systems to Generate Uniform Magnetic Field Volumes, COMSOL Conference 2010, 1-7, Boston, United States, 7-9 Oct. (2010).

Restrepo-Alvarez, A. F., Franco-Mejia, E. y Pinedo-Jaramillo, C. R., Study and analysis of magnetic field homogeneity of square and circular Helmholtz coil pairs: A Taylor series approximation, ANDESCON 2012 IEEE, 1-4, Cuenca, Ecuador, 7-9 Nov. (2012).

Torres O., J. I., Cruz Muñoz, B. y Marin Ramírez, W., Caracterización y calibración automática de bobinas de Helmholtz en DC. Scientia et Technica, XVIII(34), 535-539 (2007).

Tsz-Ka L., T., Tri-axial Square Helmholtz coil for Neutron EDM Experiment, University of Hong Kong, Hong Kong, China, 23 p. (2004).

Wang, J., She, S. y Zhang, S., An improved Helmholtz coil and analysis of its magnetic field homogeneity. Review of Scientific Instruments, 73(5), 2175-2179 (2002).

Yongshun, Z., Huaying, X., Na, W., Lvhua, W., Chunyu, D., Ying, S. y Zhuang, M., Design, analysis and experiments of a spatial universal rotating magnetic field system for capsule robot, Mechatronics and Automation (ICMA 2012), International Conference on, 998-1003, Chengdu, China, 5-8 Aug. (2012). 